\title{
Structure informationnelle et choix référentiel dans les titres de presse
}

\author{
Mathilde SALLES \\ Université de Caen Normandie \\ mathilde.salles@unicaen.fr
}

\begin{abstract}
Résumé: Dans cet article, nous cherchons à souligner l'importance des notions d'identifiabilité et d'activation (Chafe 1976) pour étudier la structure informationnelle des titres de presse et les contraintes qui pèsent sur le choix de leurs expressions référentielles. La prise en compte de ces deux notions permet une description du choix référentiel plus efficace et plus complète que la prise en compte de la seule notion d'accessibilité (Ariel 1990). Elle permet aussi d'identifier deux types de titres, dans lesquels l'expression référentielle initiale n'est pas soumise aux mêmes contraintes. Ces deux types de titres se distinguent par leur structure informationnelle: alors que les uns sont des énoncés thétiques ordinaires, les autres sont des énoncés thétiques à topique initial non lié présentant des caractéristiques comparables aux «constructions à topique non lié» de Lambrecht (1994).
\end{abstract}

\begin{abstract}
In this paper, we emphasise the importance of the notions of identification and activation (Chafe) to study the information structure of newspapers headlines and the constraints on the choice of their referential expressions. The inclusion of these two concepts enables a more efficient and comprehensive description of referential choice than taking into account the only notion of accessibility (Ariel). It also identifies two types of headlines in which the initial referential expression is not subject to the same constraints. These two types of headlines are distinguished by their informational structure: while some are ordinary thetic statements, others are thetic statements with an unlinked initial topic, similar to Lambrecht's "unlinked topic constructions".
\end{abstract}

L'objet de cet article est de souligner l'importance des notions d'identifiabilité et d'activation (Chafe 1976) pour décrire la structure informationnelle des titres de presse et pour expliquer le choix de leurs expressions référentielles. La prise en compte de ces deux catégories nous permettra non seulement, contrairement à une étude comme celle de Kronrod et Engel (2001) fondée sur la théorie de l'accessibilité d'Ariel, de rendre compte de tous les choix référentiels possibles, mais elle nous permettra aussi d'identifier deux types de titres informatifs 
dans lesquels l'expression référentielle initiale n'est pas soumise aux mêmes contraintes: des titres thétiques ordinaires (ex.: Charles Doux sort de sa coquille (Libération, 26 juillet 2012)) et des titres thétiques à topique initial non lié (ex.: Doux: la coopérative Sofiprotéol et Charles Doux vont s'affronter pour la reprise du travail (Le Monde, 26 juillet 2012)).

Après avoir soumis la théorie de l'accessibilité à l'épreuve des titres de presse - à la manière de Kronrod et Engel (2001), mais avec des résultats très différents -, on montrera que l'identifiabilité et l'activation référentielles sont deux catégories à la fois bien plus efficaces que la seule notion d'accessibilité pour expliquer le choix référentiel dans les titres de presse et décisives pour identifier leur structure informationnelle.

\section{Accessibilité référentielle et titres de presse}

Selon la théorie de l'accessibilité d'Ariel (1990), le choix d'une expression référentielle dépend du degré d'activation présumé de la représentation mentale du référent: si le locuteur présume que cette représentation est très active dans la mémoire de son auditeur/lecteur, il choisira un marqueur de forte accessibilité pour désigner le référent (par exemple un pronom personnel); si, au contraire, il estime cette accessibilité mentale faible, il utilisera un marqueur de faible accessibilité (par exemple un nom propre complet).

Quatre facteurs déterminent, chez Ariel, l'accessibilité d'un référent (ou, plus exactement, de sa représentation mentale):

- la distance entre l'expression référentielle et sa dernière mention (son antécédent): plus cette distance est courte, plus le référent est accessible;

- la compétition entre plusieurs expressions référentielles susceptibles de constituer l'antécédent: plus il y a d'antécédents possibles, moins le référent est accessible;

- la saillance du référent, c'est-à-dire sa topicalité, sa centralité dans le discours. La position syntaxique de sujet et le nombre de reprises référentielles sont des critères déterminants: plus un référent a fait l'objet de mentions, de préférence en position de sujet, plus il est accessible;

- l'unité: un référent est plus accessible si son antécédent fait partie de la même unité que son expression actuelle, autrement dit s'il fait partie du même «cadre/monde/point de vue/segment ou paragraphe» (Ariel 1990: 29). 
La relation entre les formes linguistiques et le degré d'accessibilité qu'elles codent n'est pas arbitraire. Elle répond, selon Ariel (1990: 80-83), à trois principes différents: un principe d'informativité, lié à la quantité d'information lexicale; un principe de rigidité, c'est-à-dire d'univocité, de non-ambiguïté de la forme; un principe d'atténuation, ou légèreté phonétique, en termes de longueur et d'accentuation. Moins l'expression est informative, rigide et longue, plus son référent est présumé accessible. Inversement, plus une expression est informative, univoque et longue, moins son référent est présumé accessible. Les formes codant le plus haut degré d'accessibilité seront donc les moins informatives, les plus ambiguës et les plus courtes (éléments vides, pronoms atones...) et, inversement, les formes codant le plus faible degré d'accessibilité seront les plus informatives, les plus univoques et les plus longues.

En première mention, la théorie d'Ariel prévoit l'utilisation de marqueurs de faible accessibilité, autrement dit de formes longues, informatives et univoques. Or, selon Kronrod et Engel (2001), ce qui caractériserait les titres de presse serait l'utilisation non de marqueurs de faible accessibilité, mais de formes «intermédiaires » : prénoms, noms de famille et descriptions définies courtes (i.e. descriptions définies comprenant deux unités lexicales maximum). Cette utilisation de marqueurs d'accessibilité intermédiaire serait le résultat d'un compromis entre deux tendances opposées: l'accessibilité des référents appelle des marqueurs de faible accessibilité, mais la volonté du rédacteur de ne pas prendre trop d'espace et d'éveiller la curiosité du lecteur en omettant certaines informations appelle, elle, l'emploi de formes courtes, peu informatives et peu rigides, autrement dit de marqueurs de forte accessibilité ${ }^{1}$.

Pour illustrer cette prédominance des emplois de marqueurs intermédiaires, Kronrod et Engel étudient les expressions référentielles dans les titres de trois quotidiens israéliens (en hébreu) - deux quotidiens populaires et un quotidien «de qualité» - parus le 24 janvier 1997. Elles n'observent pas de différences significatives entre ces trois quotidiens, ni entre les différentes rubriques (articles en une, sports, actualités, etc.): à chaque fois, ce sont les marqueurs d'accessibilité intermédiaire qui prédominent (entre $69,2 \%$ et $75,2 \%$ selon les quotidiens), les marqueurs

1. Comme le souligne un relecteur, la saillance contextuelle de certains référents, c'est-à-dire le fait qu'ils fassent l'actualité, pourrait aussi expliquer cette utilisation de marqueurs plus forts que ceux habituellement attendus en première mention. 
de forte accessibilité ${ }^{2}$ sont peu nombreux ( $5,6 \%$ à 9,5 \% des occurrences), sauf dans un des quotidiens populaires qui en compte $17,8 \%$, et les marqueurs de faible accessibilité - descriptions définies longues, noms propres complets et noms propres complets avec modifieur - représentent entre $13 \%$ et 19,2\% des occurrences. L'expression référentielle la plus fréquente est la description définie courte (entre $28,1 \%$ et 35,5\%), suivie de près par le prénom ${ }^{3}$ (entre $24,5 \%$ et $33,2 \%$ ), et enfin le nom de famille (entre $12,3 \%$ et $15,7 \%$ ).

Observe-t-on la même tendance à privilégier les marqueurs d'accessibilité intermédiaires dans les titres de quotidiens français? Un relevé des mêmes marqueurs d'accessibilité (moins les deux premiers marqueurs de forte accessibilité - forme zéro et accord en personne; voir le Tableau 1) dans les titres du Monde et de Libération ${ }^{4}$ du 26 juillet 2012 ne conduit pas du tout aux mêmes résultats.

Le relevé exclut plusieurs expressions référentielles, certaines rares (un pronom interrogatif dans Le Monde, un SN démonstratif dans Libération), d'autres fréquentes (des SN indéfinis ou numéraux et des SN construits sans déterminant, se rapprochant soit de descriptions définies - ex.: Mort du président Atta Mills (Le Monde) - soit de descriptions indéfinies - ex.: Arrestations aux obsèques du dissident cubain Oswaldo Paya (Le Monde)). Les expressions référentielles enchâssées n’ont pas été comptées séparément: par exemple, pour la riposte du régime de Damas (Le Monde), une seule expression référentielle, description définie longue, a été comptée et non trois, qui seraient la riposte du régime de Damas, le régime de Damas et Damas. Suivant le même principe, les déterminants possessifs n'ont pas été relevés séparément de l'expression référentielle dans laquelle ils apparaissent. Par exemple, dans le SN ses centrales nucléaires (Le Monde), une seule expression référentielle, description définie courte avec deux unités lexicales, a été prise en compte.

2. Il s'agit, dans les relevés de Kronrod et Engel, d'éléments vides, d'accords en personne (lorsqu'un nom ou un verbe contient un accord en personne; ex. : tik $+o$ $=$ «sac + son $», ~ « s o n s a c »)$ et de pronoms personnels.

3. En fait, la plupart des prénoms (ou surnoms) relevés par Kronrod et Engel sont utilisés pour référer au même individu, le Premier ministre de l'époque, Benyamin Netanyahou (souvent appelé Bibi).

4. Dans la mesure où Kronrod et Engel n'ont pas relevé de différences significatives entre leurs trois quotidiens (populaires ou de qualité), nous limitons notre étude à deux quotidiens de qualité, mais deux quotidiens dont les titres diffèrent nettement (Libération est célèbre pour ses jeux de mots). Une analyse des titres d'un autre genre de presse que la presse d'information générale, la presse à sensation (par exemple un hebdomadaire comme Le Nouveau Détective), fournirait probablement des résultats bien différents (voir infra, n. 10). 
Tableau 1. Marqueurs d'accessibilité retenus

\begin{tabular}{|c|c|c|}
\hline \multicolumn{2}{|c|}{$\begin{array}{c}\text { Marqueurs d'accessibilité } \\
\text { (par ordre d'accessibilité décroissante) }\end{array}$} & \multirow{2}{*}{$\begin{array}{l}\text { Exemples (Le Monde } \\
\text { et Libération) }\end{array}$} \\
\hline Forte accessibilité & Pronoms personnels & \\
\hline \multirow{3}{*}{$\begin{array}{l}\text { Accessibilité } \\
\text { intermédiaire }\end{array}$} & Prénoms & Nicolas $^{6}$ \\
\hline & Noms de famille & Ouattara \\
\hline & $\begin{array}{l}\text { Descriptions définies } \\
\text { courtes (jusqu’à deux } \\
\text { unités lexicales) }\end{array}$ & $\begin{array}{l}\text { l'armée; } \\
\text { les médecins; } \\
\text { les voitures propres }\end{array}$ \\
\hline \multirow{3}{*}{ Faible accessibilité } & $\begin{array}{l}\text { Descriptions définies } \\
\text { longues (trois unités } \\
\text { lexicales et plus) }\end{array}$ & $\begin{array}{l}\text { l'arsenal chimique } \\
\text { syrien; la formation } \\
\text { des prix outre-mer }\end{array}$ \\
\hline & $\begin{array}{l}\text { Noms propres com- } \\
\text { plets (prénom + nom } \\
\text { de famille, pseudo- } \\
\text { nyme, toponyme, } \\
\text { nom d'entreprise...) }\end{array}$ & $\begin{array}{l}\text { David Cameron; } \\
\text { Madonna; } \\
\text { Londres; Apple }\end{array}$ \\
\hline & 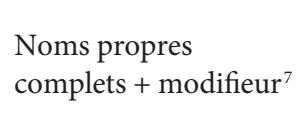 & $\begin{array}{l}\text { Rodrigo Rato, l'ancien } \\
\text { patron de Bankia; la } \\
\text { coopérative Sofiprotéol }\end{array}$ \\
\hline
\end{tabular}

C'est-à-dire que la part pronominale du déterminant possessif (d'elle dans ses centrales nucléaires) n'a pas été pas comptée séparément, de la même manière que si l'on avait eu affaire à un $\mathrm{SN}$ comme les centrales nucléaires $d^{\prime} E D F$, on n'aurait pas compté séparément le nom propre $E D F^{8}$. Les

5. Les pronoms personnels relevés sont soit des pronoms anaphoriques trouvant leur antécédent dans le titre (ex.: PSA [groupe automobile] estime qu'il doit réaliser 1,5 milliard d'euros d'économies d'ici à 2005 (Le Monde)), soit des pronoms déictiques dans une citation (ex. : En France, je n'aurais pas osé (Le Monde)).

6. C'est la seule occurrence relevée et elle est très particulière: elle est liée à un jeu de mots qui laisse apparaître le nom de famille, Tourner comme Nicolas en Cage (Libération).

7. Dans cette catégorie, on trouve à la fois des noms propres complets accompagnés d'une apposition et des noms propres complets précédés d'un nom qui précise une classe d'appartenance; en termes d'informativité, de rigidité et de longueur, on peut rapprocher les deux constructions (c'est-à-dire rapprocher Nicolas Cage, l'acteur américain et l'acteur américain Nicolas Cage).

8. De ce point de vue, la méthode de comptage adoptée ici semble différente de celle de Kronrod et Engel (qui n'est pas très explicite), puisque, on l'a vu, leur relevé inclut des formes d'accord en personne associées à un nom (voir l'exemple tik $+o=\ll \mathrm{sac}+$ son $», \ll$ son sac $»)$. 
déterminants possessifs se révèlent peu nombreux (deux occurrences dans les titres du Monde et trois dans ceux de Libération) et, à une exception près (un emploi générique dans l'expression idiomatique qui constitue le titre Abattre son joker de Libération), ils trouvent tous leur antécédent dans le titre même (ex.: EDF néglige le droit du travail dans ses centrales nucléaires (Le Monde)).

Les résultats obtenus sont, comme chez Kronrod et Engel, donnés dans deux tableaux. Le Tableau 2 présente tous les marqueurs d'accessibilité pris en considération et le Tableau 3 les rassemble en trois catégories: marqueurs de forte accessibilité (pronoms personnels), marqueurs d'accessibilité intermédiaire (prénoms, noms de famille, descriptions définies courtes) et marqueurs de faible accessibilité (descriptions définies longues, noms propres complets et noms propres complets + modifieur). Les pourcentages sont arrondis.

Dans Le Monde et Libération, l'emploi des marqueurs d'accessibilité intermédiaire est bien moins important que celui observé par Kronrod et Engel. Les pourcentages entre marqueurs d'accessibilité intermédiaire et marqueurs de faible accessibilité sont assez proches dans Libération, les marqueurs de faible accessibilité dominent dans Le Monde. Et, dans les deux cas, la proportion de marqueurs de faible accessibilité serait bien plus importante si l'on n'avait pas ignoré certaines expressions référentielles, comme les $\mathrm{SN}$ indéfinis et numéraux ou les $\mathrm{SN}$ construits sans déterminant (ceux qui se rapprochent de descriptions indéfinies - ex. : Arrestations aux obsèques du dissident cubain Oswaldo Paya - ou de descriptions définies longues - ex.: Mort du président Atta Mills marquent une accessibilité faible).

Par ailleurs, classer les descriptions définies courtes parmi les marqueurs d'accessibilité intermédiaire est souvent contestable, surtout du point de vue de la rigidité (le deuxième principe d'Ariel, lié à l'univocité de la forme). En fait, la plupart des descriptions définies courtes relevées dans les titres du Monde et de Libération sont des descriptions définies complètes (ex. : les voitures propres, avec une interprétation générique, dans L'État parie sur les voitures propres (Le Monde)), ou semi-complètes ${ }^{9}$ (ex. : L'État dans l'exemple précédent), plus courtes

9. La complétude des descriptions définies «complètes» connait des degrés, selon la capacité qu'a l'expression référentielle d'identifier seule son référent, indépendamment de tout ancrage contextuel ou non: voir la distinction chez Kleiber (1981: 250 sq.) entre l'ancrage per se que manifeste une description définie comme l'auteur des Misérables et l'ancrage contextuel que réclament des descriptions définies comme le président des États-Unis ou l'État. La première est totalement autonome, les secondes restent dépendantes de leur contexte d'énonciation (les 


\begin{tabular}{|c|c|c|}
\hline 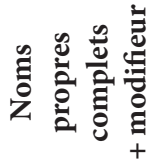 & $\stackrel{\dot{b}^{0}}{\hat{\sigma}^{\circ}}$ & 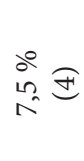 \\
\hline 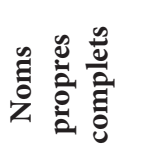 & 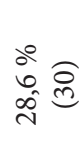 & $\begin{array}{l}\stackrel{0}{0} \\
i \mathfrak{0} \\
\dot{m}\end{array}$ \\
\hline 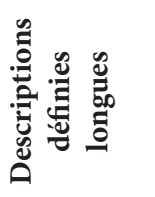 & $\begin{array}{l}\therefore \curvearrowright \\
\infty \\
=\end{array}$ & $\begin{array}{l}\sigma^{\circ} \widehat{c} \\
n_{n}\end{array}$ \\
\hline 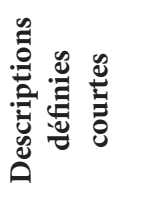 & $\begin{array}{l}\stackrel{0}{\text { J }} \\
\text { ले }\end{array}$ & $\stackrel{\circ}{\vec{m}} \stackrel{0}{=}$ \\
\hline 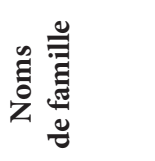 & $\begin{array}{l}a^{0} \\
\infty \\
i \\
i\end{array}$ & $\stackrel{\circ}{i n} \widehat{D}$ \\
\hline 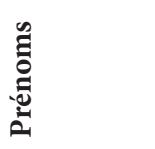 & 0 & $\stackrel{\circ}{\Rightarrow} \triangleq$ \\
\hline 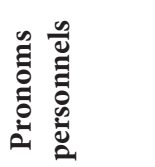 & $\begin{array}{l}\therefore 0 \\
\infty \\
\dot{n}\end{array}$ & $\stackrel{\circ}{\Rightarrow} \Xi$ \\
\hline 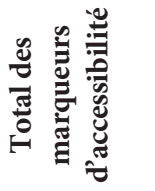 & 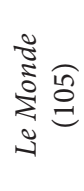 & 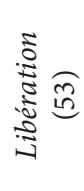 \\
\hline
\end{tabular}

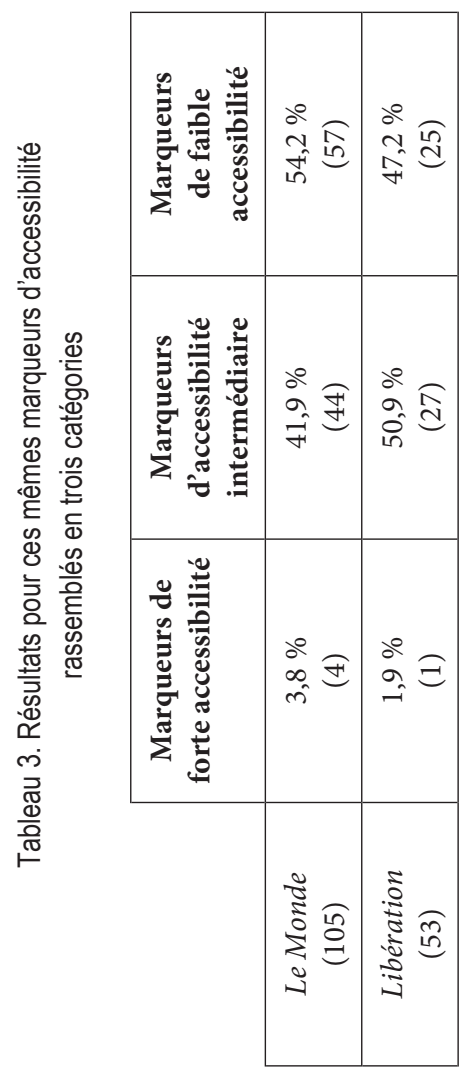


que les descriptions définies longues, mais tout aussi rigides. Elles ne marquent pas un degré d'accessibilité intermédiaire comme peuvent le faire des descriptions définies à la fois courtes et incomplètes. Distinguer les descriptions définies à la fois en fonction de leur longueur (descriptions définies courtes / descriptions définies longues; voir le premier principe d'Ariel, lié à la quantité d'information lexicale) et de leur rigidité (descriptions définies incomplètes / descriptions définies complètes; voir le deuxième principe d'Ariel) permettrait probablement une analyse plus précise des degrés d'accessibilité marqués.

Comparés aux résultats de Kronrod et Engel, nos résultats confortent donc davantage la thèse d'Ariel, en montrant que l'accessibilité du référent reste le facteur le plus important pour le choix référentiel dans les titres de presse (du moins dans ceux de quotidiens comme Le Monde et Libération $)^{10}$, et n'appuient pas la thèse du compromis censé résoudre le conflit entre d'une part l'accessibilité du référent et d'autre part la nécessité de brièveté et d'accroche des titres de presse.

\section{Identifiabilité et activation référentielles dans les titres de presse}

Si l'accessibilité reste un facteur décisif pour le choix référentiel dans les titres de quotidiens comme Le Monde et Libération, elle ne permet toutefois pas de rendre compte de tous les choix référentiels. Selon Chafe (1994: 179; nous traduisons), «bien qu'Ariel mentionne différents

référents visés ne peuvent être identifiés sans une prise en compte de leur contexte d'énonciation, qui leur fournit l'ancrage - temporel pour le président des ÉtatsUnis, temporel et locatif pour l'État - nécessaire). La première est donc plus rigide au sens d'Ariel que les secondes, et, comme on le constate, ce n'est pas forcément une affaire de longueur.

10. La presse à sensation opère des choix référentiels très différents, comme on peut le constater dans les titres de France Dimanche, Ici Paris, Allo Police et Qui? Police relevés par Charaudeau (1983: 102). Ces titres présentent un grand nombre de marqueurs de forte accessibilité et d'accessibilité intermédiaire, pronoms de 3 personne sans antécédent et «vraies» descriptions définies courtes, c'est-à-dire incomplètes: Il utilise la magie noire pour abuser des femmes, Il exécute son rival en l'opérant, Le satyre avait peur des femmes. L'utilisation d'un marqueur d'accessibilité forte ou intermédiaire pour un référent qui n'est pas encore accessible produit un effet d'accroche bien plus spectaculaire que celui évoqué par Kronrod et Engel (2001), un effet qu'on pourrait décrire à la manière de Tom Wolfe: «Pensez donc à ce superbe sens de la syntaxe du caniveau qui les avait inspirés pour créer un titre fait de verbes et d'objets, où le vrai sujet manquait, meilleur moyen de vous faire plonger vos griffes dans ces pages noires et grasses pour savoir quel démon était assez monstrueux pour être ce "IL" (Le bûcher des vanités, B. Legrand (trad.), Paris, Librairie générale française (Le Livre de poche), 2013, p. 229-230). 
degrés de “disponibilité mémorielle”, ce qui est réellement en jeu dans sa théorie, c'est la nature du langage nécessaire pour rendre un référent partagé identifiable dans un contexte donné». Son échelle, qui va des expressions qui réclament le plus d'information verbalisée (les noms propres complets + modifieur) pour la bonne identification d'un référent partagé à celles qui ne réclament pas d’information verbalisée (les éléments vides), ordonne ainsi des expressions aux référents identifiables (Ariel 1990: 73). Et les expressions indéfinies, dont le référent n'est pas toujours identifiable (il ne l'est pas lorsqu'elles reçoivent une interprétation spécifique existentielle), n'ont pas leur place dans cette échelle, alors qu'elles constituent une part relativement importante des expressions qu'on peut relever dans les titres de presse.

Il existe, en outre, sur l'emploi de ce type d'expressions référentielles, des contraintes que la théorie de l'accessibilité est incapable d'expliquer. La prise en compte de deux catégories de la structure informationnelle, l'identifiabilité du référent et son activation (Chafe 1976 et 1994), permet, au contraire, de bien distinguer les facteurs qui déterminent le choix référentiel dans les titres de presse. Ces deux catégories sont définies ainsi par Lambrecht (1994: 76 et 94):

- l'identifiabilité concerne l'évaluation par un locuteur de la présence ou non dans l'esprit de l'auditeur d'une représentation de discours du référent. Un référent identifiable est un référent pour lequel il existe déjà une représentation partagée par le locuteur et l'auditeur au moment de l'acte de parole, alors qu'un référent non identifiable est un référent dont seul le locuteur a une représentation;

- l'activation concerne l'évaluation par un locuteur du statut de la représentation d'un référent identifiable comme déjà activée, simplement accessible ou inactive dans l'esprit de l'auditeur au moment de l'acte de parole. La représentation d'un référent est active lorsqu'elle est actuellement «allumée» dans l'esprit de l'auditeur, pour reprendre la métaphore de Chafe. Elle est accessible (ou semi-active) lorsqu'elle n'est pas directement dans le focus d'attention, mais en arrière-plan, dans une zone périphérique de la conscience, et inactive lorsqu'elle est disponible dans la mémoire à long terme seulement.

La forme des éléments initiaux des titres de presse est conditionnée par deux caractéristiques ayant des incidences l'une sur l'identifiabilité du référent, l'autre sur son activation. Leur statut généralement non topical permet l'utilisation d'expressions aux référents non identifiables pour le lecteur, sans représentation déjà disponible dans l'esprit du lecteur. Le fait qu'elles constituent généralement la toute première mention 
d'un référent a pour corollaire le fait que, si le référent en question est identifiable, il est alors inactif. Sauf cas de reprise à l'intérieur même du titre (ex.: PSA estime qu'il doit réaliser 1,5 milliard d'euros d'économie d'ici à 2005 (Le Monde)), on ne trouvera donc guère d'expressions codant le statut actif comme les pronoms personnels dans les titres de presse (presse à sensation exceptée, comme nous l'avons vu avec les titres de la note 10).

\subsection{Statut non topical des expressions référentielles dans les titres de presse}

Les titres de presse sont des énoncés thétiques, c'est-à-dire des énoncés dont la « raison d'être au niveau discursivo-pragmatique [...] est de servir à présenter une entité, une proposition ou un état de choses en tant qu'élément d'information nouveau pour le discours» (Cornish 2008: 122). Lorsque les titres présentent une structure sujet-prédicat, le sujet fait donc partie du contenu asserté, non présupposé, c'est-à-dire du focus; il n'est pas le topique ${ }^{11}$. Le topique, ce dont on parle et ce dont on accroît la connaissance ${ }^{12}$, est simplement ce qui est arrivé aujourd'hui.

Dans la mesure où il n'est pas le topique, le sujet n'est soumis à aucune contrainte référentielle: contrairement aux référents en position de topique ${ }^{13}$, son référent n'exige pas d'être identifiable. On peut alors aussi bien trouver des $\mathrm{SN}$ indéfinis spécifiques à lecture existentielle (exemples (1)), dont les référents ne sont pas encore identifiables pour le lecteur, que des SN définis ou des noms propres (exemples (2)) aux référents identifiables, en position de sujet ${ }^{14}$ :

11. Ce qui caractérise les phrases thétiques, selon Lambrecht (1994: 144-145), ce n'est pas l'absence de topique, mais l'absence de sujet-topique: s'il y a un sujet grammatical, celui-ci n'est pas un topique, mais fait partie du focus.

12. Voir à cet égard la définition de Lambrecht (1994: 127; nous traduisons): «Un référent est interprété comme le topique d'une proposition si dans un discours donné la proposition est interprétée comme étant à propos de ce référent, i.e. comme exprimant une information qui est pertinente pour ce référent et qui accroît la connaissance qu'a l'interlocuteur de ce référent».

13. Selon Gundel (1985), en effet, l'expression référant au topique doit être définie ou générique, c'est-à-dire qu'il doit s'agir d'une expression au référent identifiable pour l'auditeur / lecteur. Dans les langues qui, comme le japonais, ont un marqueur de topicalité, celui-ci ne peut apparaitre qu'avec un SN dont le référent est défini ou générique; de même, la dislocation à droite, structure généralement associée au marquage du topique, n'autorise que des $\mathrm{SN}$ à référence définie ou générique (voir Gundel 1985: 91).

14. Fréquemment dans des structures elliptiques, faisant l'économie de l'auxiliaire (1.a) ou du verbe attributif être (1.b). 
1. a. Une nouvelle crèche clandestine découverte à Marseille. (Le Monde, 26 juillet 2012)

b. Des archéologues italiens sur les traces de «la Joconde» à Florence. (Le Monde, 26 juillet 2012)

2. a. L'arsenal chimique syrien alarme Israël. (Libération, 26 juillet 2012)

b. Le gouvernement fait le pari de l'automobile verte. (Le Monde, 26 juillet 2012)

c. Charles Doux sort de sa coquille. (Libération, 26 juillet 2012)

Les SN des titres de presse, quelle que soit leur position syntaxique, ne posent-ils pour autant aucune contrainte quant à l'identifiabilité de leurs référents?

\subsection{Un autre type de titre: des énoncés thétiques à topique initial}

On observe une structure récurrente dans les titres de presse qui semble exiger une identifiabilité du référent initial. Cette structure, dite «bisegmentale» par Bosredon et Tamba $(1992)^{15}$, présente un SN initial suivi de deux-points introduisant soit une structure phrastique complète ou à ellipse du verbe être (3), soit un SN ou des SN coordonnés $(4)^{16}$ :

3. a. Doux: la coopérative Sofiprotéol et Charles Doux vont s'affronter pour la reprise du travail. (Le Monde, 26 juillet 2012)

b. Salaires: Bailly suit le décret à la lettre. (Libération, 26 juillet 2012)

c. Dépassements d'honoraires: les médecins sur la défensive. (Le Monde, 26 juillet 2012)

15. Cette structure fait partie du type «parataxe» dans la typologie de Sullet-Nylander (1998). En plus du type de titres illustré par (3)-(4), le type parataxe comprend des structures appositives telles que Ri Sol-ju, première dame coréenne (Libération, 26 juillet 2012) ou de juxtaposition coordonnante comme dans Madonna, ô Graal, ô désespoir (Libération, 26 juillet 2012 : exemple dans lequel on pourrait remplacer la virgule après Madonna par la conjonction ou), types de titres qu'on n'évoquera pas ici.

16. Dans ce dernier cas, on peut trouver une virgule à la place des deux-points: $J O$, Londres triple vainqueur (Le Monde, 26 juillet 2012), PSA-Montebourg, beaucoup de cris pour rien (Libération, 26 juillet 2012). 
4. a. Nucléaire: les infractions d'EDF au droit du travail. (Le Monde, 26 juillet 2012)

b. Cour de justice de la République: sept PS, quatre UMP et un centriste. (Le Monde, 26 juillet 2012)

Ces énoncés sont bien thétiques dans la mesure où leur objet est de présenter «une proposition ou un état de choses en tant qu'élément d'information nouveau pour le discours » (voir Cornish 2008: 122, déjà cité). Conformément à la caractérisation des énoncés thétiques faite par Lambrecht (1994), s'il y a un sujet grammatical (exemples (3)), celui-ci n'est pas un topique, mais fait partie du focus. Il n'est donc pas soumis à la contrainte d'identifiabilité et pourrait être réalisé, même si ce n'est pas le cas dans les exemples relevés, par des SN indéfinis ou numéraux à lecture existentielle; par exemple:

5. a. Doux: une offre de reprise (a été) déposée par Sofiprotéol.

b. Cour de justice de la République: sept PS, quatre UMP et un centriste jugeront leurs pairs.

En revanche, de tels $\mathrm{SN}$ semblent exclus de la position initiale de ces énoncés. Tous les $\mathrm{SN}$ initiaux de ces titres ont des référents identifiables. Seule cette contrainte d'identifiabilité pèse sur le type de SN pouvant être topique, ce qui n'exclut pas les SN sans déterminant de (3.b-c)-(4) (auxquels Bosredon et Tamba (1992:37) refuseraient pourtant le statut de thème, en partie à cause de cette forme non déterminée). Deux types de SN sont utilisés: des noms propres ou, le plus souvent, des SN construits sans déterminant. Aucun de ces SN sans déterminant n’a une référence indéfinie.

Il n'est pas impossible d'imaginer des titres apparemment proches des précédents avec un SN numéral initial:

6. a. Deux concurrents pour Doux : la coopérative Sofiprotéol et Charles Doux vont s'affronter pour la reprise du travail.

b. Douze élus à la Cour de justice de la République: sept PS, quatre UMP et un centriste.

Mais la structure informationnelle de ces titres-là se révèle très différente de celle des précédents : les éléments initiaux de (6) constituent clairement le focus, le contenu asserté, la suite (après les deux-points) ne faisant que préciser quels sont les deux concurrents pour l'avenir de Doux, quels sont les douze élus à la Cour de justice de la République. 
Ces éléments initiaux pourraient constituer le titre à eux seuls. Dans les titres présentés en (3)-(4), en revanche, ce qui suit l'élément initial ne constitue pas une spécification de ce qui précède et l'élément initial ne saurait constituer à lui seul le titre. Il ne pourrait à lui seul recevoir une interprétation thétique et ne saurait répondre à une question comme Qu'est-il arrivé? / Que s'est-il passé? / Quoi de neuf?

7. Qu'est-il arrivé? / Quoi de neuf?

a. (Il y a) Deux concurrents pour Doux.

b. \# Doux.

En fait, si l'on devait utiliser le test question-réponse pour identifier le focus des énoncés présentés en (3)-(4), une question comprenant l'élément initial conviendrait davantage, la réponse se limitant à la suite du titre:

8. Quoi de neuf à propos de Doux/ des dépassements d'honoraires?

a. (À propos de Doux,) la coopérative Sofiprotéol et Charles Doux vont s'affronter pour la reprise du travail.

b. (À propos des dépassements d'honoraires,) les médecins sont sur la défensive.

L'élément initial constitue une forme de rubrique ${ }^{17}$ pour ce qui suit et c'est aussi une entité, un événement ou un état de choses dont le lecteur a déjà une représentation (un sujet de société ou un sujet qui fait l'actualité du moment), autrement dit identifiable. Le test question-réponse souligne le statut topical de ces éléments initiaux: ce dont on parle, c'est de l'entreprise Doux et ce qui suit est bien «à propos de ce référent», exprime bien « une information qui est pertinente pour ce référent et qui accroît la connaissance qu'a l'interlocuteur de ce référent», conformément à la définition donnée par Lambrecht (1994: 127; nous traduisons).

Si la paraphrase en à propos de proposée en (8.a-b) peut être considérée comme une mise en relief du topique, alors ces SN initiaux sont bien des formes de topiques. Cette paraphrase rappelle les tests d'identification du topique en as for et about en anglais (voir, entre autres, Kuno 1972, Reinhart 1981, Lambrecht 1994, Gundel \& Fretheim 2004) et en quant à en français. Mais, contrairement à ces tests (9.a-c), l'élément introduit par à propos de n'est pas repris dans la suite de l'énoncé:

17. Fonction de pseudo-rubrique selon Bosredon et Tamba (1992: 42), qui proposent eux-mêmes une glose en à propos de ou en ce qui concerne. 
9. a. As for Rosa, John didn’t really love her. (Lambrecht 1994: 149)

b. John said about Rosa that he didn't really love her. (Lambrecht 1994: 149)

c. Quant à Rosa, John ne l'aime pas vraiment.

En outre, alors que à propos de semble pouvoir être un simple introducteur de topique, le détachement en quant à indique un changement de topique parmi un ensemble de topiques déjà disponibles, ce qui suppose un contexte discursif antérieur et explique que l'expression référentielle introduite par quant $\grave{a}$, comme celle du as-for test, doive avoir un référent non seulement identifiable (comme tout topique), mais aussi un référent qui ne soit pas inactif. Comme le rappelle Lambrecht (1994: 152; nous traduisons),

[...] le syntagme As for $S N$ (tout comme des syntagmes similaires dans d'autres langues) ne peut être utilisé de façon appropriée que si le référent du SN est déjà un topique potentiel du discours au moment où ce syntagme est utilisé, autrement dit lorsque ce référent est contextuellement accessible.

Ainsi, l'énoncé As for your brother, I saw him last night, ajoute Lambrecht (1994: 152; nous traduisons), «n'est approprié que si le frère appartient à l'ensemble des référents dont on discute». On peut dire la même chose d'un énoncé comme Quant à ton frère, je l'ai vu hier soir.

Nos paraphrases en à propos de ne posent pas ce type de contraintes: dans la mesure où elles n'indiquent pas un changement de topique parmi un ensemble de topiques déjà disponibles et ne supposent pas ainsi un discours préalable, elles n'exigent pas un statut d'activation particulier du référent. Ce dernier a seulement besoin, en tant que topique, d'être identifiable, mais il peut très bien être inactif. Il l'est dans la plupart de nos titres ${ }^{18}$.

18. Pas dans tous: certains titres constituent des reprises de titres de la une; lors de leur nouvelle mention, les référents peuvent être considérés comme semi-actifs (Lambrecht 1994: 99-100), plus précisément comme "accessibles textuellement» (Lambrecht 1994: 100, parle de référents «accessibles textuellement» dans les cas de désactivation d'un état antérieur actif dans le discours). C'est le cas, par exemple, du référent associé au SN initial du titre Honoraires libres: pourquoi les médecins résistent (Le Monde, 26 juillet 2012, p. 7), forme de reprise du SN initial du titre antérieur Dépassements d'honoraires: les médecins sur la défensive (Le Monde, 26 juillet 2012, p. 1). 
Ces topiques, à l'initiale d'un énoncé thétique, répondent bien à la définition de topique que donne Chafe (1976), en termes d'expression «scene setting», c'est-à-dire d'expression qui installe « un cadre spatial, temporel ou individuel dans lequel a lieu la prédication principale» et qu'il distingue du «sujet», caractérisé comme «point d'ancrage pour la nouvelle connaissance» (Chafe 1976, cité par Lambrecht 1994: 118; nous traduisons). Selon Lambrecht, c'est une façon pour Chafe de distinguer les topiques qui ne sont pas intégrés syntaxiquement et sémantiquement dans la structure prédicat-argument(s) d'une proposition de ceux qui le sont. La notion de topique qu'utilise Lambrecht recouvre, quant à elle, ces deux types de topiques (arguments ou non). Les référents des expressions nominales initiales des exemples (3)-(4) sont interprétés comme des topiques dans la mesure où ce qui suit est bien à propos d'eux.

Ce type de titre peut être rapproché des « constructions à topique non lié» («unlinked topic constructions») de Lambrecht (1994: 193), constructions, fréquentes à l'oral, dans lesquelles un élément nominal est détaché sans être repris par un élément pronominal dans la proposition qui suit:

10. a. Tulips, you have to plant new bulbs every year? (Lambrecht 1994: 193)

b. La mer, tu vois de l'eau. (Lambrecht 1998: 42)

c. - Je vais acheter le matelas.

- Le matelas/ je veux aussi venir. (Berrendonner \& Reichler-Béguelin 1997: 205)

On en trouve aussi quelques exemples à l'écrit, en latin, avec certains cas de «nominativus pendens»:

11. Altera nihil obstat. («L'autre, rien ne fait obstacle»; Horace, relevé par Serbat 1991:25)

Les titres (3)-(4) seraient ainsi des formes de constructions à topique non lié, propres cette fois non à l'oral, mais aux titres journalistiques. Leurs expressions nominales initiales ont la même fonction cadrative que certaines des expressions initiales détachées évoquées par Charolles (1997), des expressions comme à propos de X, au sujet de X, concernant $X$, pour ce qui est de $X^{19}$ : elles instaurent des cadres que Charolles (1997: 26) appelle «champs thématiques».

19. Expressions que Charolles (1997: 26, n. 23) compare d'ailleurs au «nominativus pendens» en latin. 


\section{Conclusion}

Les relevés des expressions référentielles dans les titres du Monde et de Libération confortent assez bien la thèse d'Ariel: on trouve majoritairement des marqueurs de faible accessibilité, surtout lorsqu'on élargit cette catégorie à d'autres marqueurs, comme la plupart des descriptions définies courtes. Kronrod et Engel (2001) traitent uniformément les descriptions définies courtes comme des marqueurs d'accessibilité intermédiaire, alors que la plupart de celles qu'on peut relever dans les titres de presse sont tout aussi rigides et univoques que des descriptions définies plus longues.

La théorie de l'accessibilité référentielle ne permet cependant pas de rendre compte de tous les choix référentiels possibles: il n'y a pas de place dans l'échelle d'accessibilité pour les formes spécialisées dans les mentions initiales (les indéfinis existentiels), qui constituent une part non négligeable des expressions référentielles relevées dans les titres de presse et qui sont par ailleurs exclues de la position initiale de certains titres. La prise en compte des deux catégories de Chafe (1976), l'identifiabilité et l'activation référentielles, permet, au contraire, de décrire l'ensemble des choix référentiels possibles et de souligner des différences de contraintes selon la structure informationnelle des titres - thétiques ordinaires ou thétiques à topique initial non lié.

Les titres thétiques ordinaires n'imposent aucune restriction sur l'identifiabilité des référents et privilégient l'emploi d'expressions dont les référents sont inactifs. Hormis quelques cas de reprises à l'intérieur même d'un titre, rares seront les formes habituellement associées aux référents actifs comme les pronoms personnels.

Les titres à topique initial non lié imposent, eux, une contrainte d'identifiabilité sur le premier référent. Celui-ci doit, en effet, être identifiable, contrainte généralement admise pour les topiques. Et, dans la plupart des cas, il sera lui aussi inactif. Cette contrainte d'identifiabilité exclut de la position initiale les $\mathrm{SN}$ indéfinis qu'on peut trouver à l'initiale des titres thétiques ordinaires (ex.: Des archéologues italiens sur les traces de «la Joconde» à Florence (Le Monde, 26 juillet 2012)). Une construction avec un $\mathrm{SN}$ indéfini initial comme Des archéologues italiens: sur les traces de «la Joconde» à Florence ne semble pas acceptable. Et un titre comme Archéologues italiens: sur les traces de «la Joconde» à Florence supposerait une identifiabilité du référent, une référence définie introduisant un nouvel épisode du feuilleton des archéologues italiens et de leur quête du modèle de la Joconde. 


\section{Références bibliographiques}

Ariel M. (1990), Accessing Noun-Phrase Antecedents, Londres - New York, Routledge.

Berrendonner A., Reichler-Béguelin M.-J. (1997), «Left Dislocation in French: Varieties, Norm and Usage», Taming the Vernacular: From Dialect to Written Standard Language, J. Cheshire, D. Stein (dir.), Londres - New york, Longman, p. 200-217.

Bosredon B., Tamba I. (1992), "Thème et titre de presse: les formules bisegmentales articulées par un "deux points" ", L'information grammaticale, no 54 , p. 36-44.

Chafe W. (1976), «Givenness, Contrastiveness, Definiteness, Subjects, Topics, and Point of View», Subject and Topic, C. Li (dir.), New York San Francisco - Londres, Academic Press, p. 25-55.

Chafe W. (1994), Discourse, Consciousness, and Time, Chicago - Londres, The University of Chicago Press.

Charaudeau P. (1983), Langage et discours. Éléments de sémiolinguistique, Paris, Hachette.

Charolles M. (1997), "L'encadrement du discours: univers, champs, domaines et espaces", Cahier de recherche linguistique, $\mathrm{n}^{\circ}$ 6, p. 1-73.

Cornish F. (2008), «L'absence de prédication, le topique et le focus: le cas des phrases thétiques », Faits de langue, no 31-32, p. 121-131.

Gundel J. (1985), " "Shared Knowledge” and Topicality», Journal of Pragmatics, vol. 9, no 1, p. 83-107.

Gundel J., Fretheim T. (2004), «Topic and Focus», The Handbook of Pragmatics, L. R. Horn, G. L. Ward (dir.), Malden, Blackwell, p. 175-196.

KLeiber G. (1981), Problèmes de référence: descriptions définies et noms propres, Paris, Klincksieck.

Kronrod A., Engel O. (2001), «Accessibility Theory and Referring Expressions in Newspaper Headlines», Journal of Pragmatics, vol. 33, $\mathrm{n}^{\circ} 5$, p. 683-699.

Kuno S. (1972), «Functional Sentence Perspective: A Case Study from Japanese and English", Linguistic Inquiry, vol. 3, n 3, p. 269-320.

LAmbrecht K. (1994), Information Structure and Sentence Form, Cambridge - New York - Melbourne, Cambridge University Press.

LAMBRECHт K. (1998), «Sur la relation formelle et fonctionnelle entre topiques et vocatifs ", Langues, $\mathrm{n}^{\circ}$ 1, p. 34-45.

Reinhart T. (1981), «Pragmatics and Linguistics: An Analysis of Sentence Topics», Philosophica, n² 27, p. 53-94. 
Serbat G. (1991), «Intégration à la phrase latine d'un groupe nominal sans fonction syntaxique (le "nominativus pendens") ", Langages, n 104 , p. 22-32.

Sullet-Nylander F. (1998), Le titre de presse. Analyses syntaxique, pragmatique et rhétorique, Stockholm, Institutionen för franska och italienska, Stockholm Universitet. 